\title{
Comparación midazolam vs dexmedetomidina en pacientes con alto riesgo de síndrome de apnea obstructiva del sueño
}

\author{
Becher N. ${ }^{1,2}$, Urchipía C. ${ }^{1,2}$, García V. ${ }^{1,2}$, Glavinovich I. ${ }^{1,2}$, Harvey G. ${ }^{1,3}$, Soto G. ${ }^{1,2}$ \\ 1 Carrera Posgrado Esp en Anestesiología - FCM-UNR, Rosario, Argentina. \\ 2 Hospital Escuela Eva Perón, Gro Baigorria, Argentina. \\ 3 Fundación Anestesiológica de Rosario, Rosario, Argentina.
}

Introducción: El Síndrome de Apnea Obstructiva del Sueño (SAOS) se asocia con incremento perioperatorio de la morbimortalidad, debido a complicaciones por sedantes y opioides, recomendándose su detección precoz mediante cuestionario STOP-Bang. Pacientes STOP-Bang positivos son considerados de alto riesgo de SAOS, sugiriéndose control estricto de la ventilación y profundidad de hipnosis. Midazolam es ampliamente utilizado para sedación, sin embargo, sus efectos se asocian a relajación de la vía aérea, episodios obstructivos, hipercapnia e hipoxia. En contraposición, dexmedetomidina se relaciona con falta de depresión respiratoria y reducción del consumo de opioides.

Objetivos: Comparar efecto de sedación, midazolam versus dexmedetomidina, en funciones hemodinámicas, respiratorias y profundidad de hipnosis, en pacientes STOP-Bang positivo bajo anestesia espinal.

Métodos: Estudio clínico prospectivo, doble ciego, aleatorizado, sesenta pacientes bajo bloqueo espinal y sedación. Grupo MDZ ( $\mathrm{n}=29)$ : midazolam 0,05 mg/Kg; Grupo DEX ( $=29)$ : dexmedetomidina $0,4 \mathrm{ug} / \mathrm{kg} / \mathrm{h}(\mathrm{IC})$. Se evaluaron parámetros hemodinámicos (TAS, TAD y FC), ventilatorios (FR, SAT O2, ETCO2), profundidad de hipnosis (BIS), en períodos basal, 5, 10, 15, 30, 45 y 60 minutos. Criterios de inclusión: pacientes ASA I y II, 18 y 60 años, STOP-Bang positivos ( +3 criterios positivos), ambos sexos. Criterios de exclusión: contraindicación de bloqueo central, alergia conocida a las drogas, negativa del paciente. Análisis estadístico: datos cualitativos se expresan como $\mathrm{n}^{\circ}$ (\%), se utilizó test Chi-cuadrado y test de Fisher. Datos cuantitativos se expresan como promedio (desvío estándar), se utilizó test t. Nivel de significación: 0,05.

Resultados: Datos demográficos similares en ambos grupos (Tabla 1). Sin diferencia significativa entre grupos respecto TAS, TAD y FC. El grupo DEX tuvo valores mayores de FR (X \pm de) 5\&apos; 14,4 $\pm 2,0$ vs 12,3 $\pm 2,9$ (p $<0,0001), 10 \&$ apos; $14,3 \pm 2,2$ vs $11,0 \pm 3,0$ ( $\mathrm{p}<0,0001), 15 \&$ apos; $14,0 \pm 2,2$ vs $11,3 \pm 3,0$ ( $\mathrm{p}=0,001), 30$ \&apos; $13,9 \pm 2,0$ vs $11,5 \pm 2,9(\mathrm{p}=0,001), 45 \&$ apos; $13,8 \pm 1,8$ vs $11,6 \pm 2,7(\mathrm{p}=0,0019), 60 \&$ apos; $13,7 \pm 1,7$ vs 11,7 \pm $2,6(\mathrm{p}=0,001),($ Figura 1) y SAT O2 5\&apos; $(\mathrm{p}=0,032), 10 \&$ apos; $(\mathrm{p}=0,033), 15 \&$ apos; $(\mathrm{p}=0,059), 30 \&$ apos; $(\mathrm{p}$ $=0,033), 45 \&$ apos; $(p=0,047), 60 \&$ apos; $(p=0,028)$ comparado con MDZ. El grupo DEX menor ETCO2 10\&apos; $(\mathrm{p}=0,013)$ respecto a MDZ. El grupo DEX valores mayores de BIS 5\&apos; 91,5 $\pm 4,3$ vs 83,6 $\pm 7,2(\mathrm{p}<0,0001)$, 10\&apos; $89,0 \pm 3,2$ vs $81,3 \pm 5,1$ ( $<<0,0001$ ), 15\&apos; 87,6 $\pm 3,3$ vs $81,6 \pm 5,1$ (p = 0,001), 30\&apos; 87,8 $\pm 4,2$ vs $82,3 \pm 4,2(\mathrm{p}<0,001), 45 \&$ apos; $87,5 \pm 5,4$ vs $83,9 \pm 5,1(\mathrm{p}=0,003), 60 \&$ apos; $88,6 \pm 4,5$ vs $86,4 \pm 5,8(\mathrm{p}=$ 0,038), comparado con MDZ (Figura 2).

Conclusión: La sedación con dexmedetomidina en pacientes STOP Bang positivo bajo anestesia espinal, proporciona estabilidad hemodinámica, con mejor control de parámetros respiratorios y profundidad de hipnosis, en comparación con midazolam.

https://doi.org/10.25237/congresoclasa2019.09 